\title{
Association of polymorphisms in the heparanase gene (HPSE) with hepatocellular carcinoma in Chinese populations
}

\author{
Lixia $\mathrm{Yu}^{1}$, Xiaoai Zhang ${ }^{2}$, Yun Zhai ${ }^{2}$, Hongxing Zhang ${ }^{2}$, Wei Yue ${ }^{2}$, Xiumei Zhang ${ }^{2}$, Zhifu Wang ${ }^{2}$, \\ Hong Zhou', Gangqiao Zhou ${ }^{2}$ and Feng Gong ${ }^{1}$ \\ ${ }^{1}$ Institute of Transfusion Medicine, Beijing, P. R. China. \\ ${ }^{2}$ State Key Laboratory of Proteomics, Beijing Proteome Research Center, Beijing Institute of Radiation \\ Medicine, Beijing, P. R. China.
}

\begin{abstract}
Heparanase activity is involved in cancer growth and development in humans and single nucleotide polymorphisms (SNPs) in the heparanase gene (HPSE) have been shown to be associated with tumors. In this study, we investigated whether SNPs in HPSE were a risk factor for hepatocellular carcinoma (HCC) by undertaking a comprehensive haplotype-tagging, case-control study. For this, six haplotype-tagging SNPs (htSNPs) in HPSE were genotyped in $400 \mathrm{HCC}$ patients and 480 controls by polymerase chain reaction-restriction fragment length polymorphism (PCR-RFLP) analysis. A log-additive model revealed significant correlations between the HPSE polymorphisms rs12331678 and rs 12503843 and the risk of HCC in the overall samples $(p=0.0046$ and $p=0.0055)$. When the analysis was stratified based on hepatitis B virus (HBV) carrier status, significant interactions between rs 12331678 and rs12503843 and HBV were observed. Conditional logistic regression analysis for the independent effect of one significant SNP suggested that rs 12331678 or rs 12503843 contributed an independent effect to the significant association with the risk of HCC, respectively. Our findings suggest that the SNPs rs 12331678 and rs 12503843 are HCC risk factors, although the potential functional roles of these two SNPs remain to be fully elucidated.
\end{abstract}

Keywords: genetic association, heparanase, hepatocellular carcinoma, HPSE gene, PCR-RFLP.

Received: November 18, 2014; Accepted: May 11, 2015.

\section{Introduction}

Human hepatocellular carcinoma (HCC), one of the most common tumors in the world, has a high incidence in China, Southeast Asia and sub-Saharan Africa, but a low incidence in the United States and Europe (Ferlay et al., 2010). Malignant growth and metastasis are key features of $\mathrm{HCC}$ and are associated with a poor prognosis (Schafer and Sorrell, 1999). During metastasis, tumor cells penetrate the extracellular matrix (ECM) and basement membrane (BM) (Sasisekharan et al., 2002), but adhere poorly to each other; detachment from neighboring cells facilitates entry into blood and lymphatic vessels and dissemination to distant organs.

Heparanase, a mammalian endo- $\beta$-D-glycosidase, specifically cleaves the heparan sulphate side chains of heparin sulfate glycosaminoglycans, the most abundant macromolecules in the basement membrane (BM) and ECM (Sasisekharan et al., 2002). Heparanase activity can influence a number of normal and pathological processes, includ-

Send correspondence to Feng Gong. Institute of Transfusion Medicine, 27 Taiping Road, Beijing, 100850, P. R. China E-mail: gongfeng@nic.bmi.ac.cn ing tissue repair, inflammation, tumor growth, metastasis and angiogenesis (Bishop et al., 2007). In the liver, heparin sulphate-degrading activity is also involved in normal and pathological processes, such as liver development, remodeling and malignant growth (Goldshmidt et al., 2004).

Various studies have examined the clinical significance of heparanase in HCC patients using immunohistochemistry, in situ hybridization, RT-PCR and real timePCR, western blotting and tissue microarrays (TMAs), with the general conclusion being that heparanase is upregulated in HCC (El-Assal et al., 2001; Xiao et al., 2003; Chen et al., 2004; Liu et al., 2005; Chen et al., 2008). Down-regulating heparanase expression using either antisense oligodeoxynucleotides or RNA interference significantly inhibits the invasiveness, metastasis and angiogenesis of human HCC SMMC7721 cells in vitro and in vivo (Zhang et al., 2007). Two anti-heparanase antibodies (multiple antigenic peptides MAP1 and MAP2) can effectively inhibit the heparanase activity of HCCLM6 liver cancer cells, thereby influencing their invasive capacity (Yang et al., 2009). Together, these findings indicate that heparanase plays a vital role in HCC metastasis and tumor growth. 
The heparanase gene (HPSE), located on chromosome 4q21.3, was first cloned in 1999. Several previous reports suggested that single nucleotide polymorphisms (SNPs) in HPSE are associated with various types of cancers, including ovarian carcinoma, hematological malignancies, and gastric cancer (Ostrovsky et al., 2007; Ralph et al., 2007; Winter et al., 2008; Yue et al., 2010; Huang et al., 2012; Li et al., 2012). Huang et al. (2012) demonstrated that allele loss and reduced HPSE expression are closely correlated with tumor progression and poor prognosis in HCC patients. HPSE is a tumor suppressor gene based on the fact that tumor suppressor genes usually cause loss of heterozygosity (LOH) in carcinogenesis (Huang et al., 2012). The role of HPSE in HCC is currently controversial. In this study, we selected six haplotype-tagging SNPs (htSNPs) distributed throughout the entire gene and investigated whether polymorphisms in HPSE were associated with the risk of HCC in a Chinese population.

\section{Materials and Methods}

\section{Subjects}

This case-control study consisted of 400 incident patients with $\mathrm{HCC}$ and 480 healthy controls. All subjects were Chinese recruited in Fusui territory and surrounding regions in Guangxi province, a high-risk region for HCC in southern China. The diagnosis of cases, the inclusion and exclusion criteria, the definition of hepatitis B virus (HBV) carriers, smokers and drinkers were as described previously (Zhai et al., 2006, 2007). Thirty-four of the original 434 cases were excluded because genomic DNA was depleted in the original study (Zhang et al., 2010); the remaining 400 cases were included in the study (Table 1). There were no significant differences between the initial 434 and final 400 patients in terms of age and sex distributions (mean ages: 49.1 and 49.3 years, respectively; male/female ratio: 6.6 and 6.5 , respectively). Informed consent was obtained from each subject at recruitment and personal information on demographic factors, medical history, tobacco and alcohol use and family history of HCC was collected via a structured questionnaire. This study was approved by the Medical Ethical Committee of the Chinese National Human Genome Center.

\section{Selection of haplotype-tagging SNPs (htSNPs)}

The htSNPs and candidate SNPs were selected to allow complete HPSE gene coverage and to replicate previously reported associations. The htSNPs were selected from genotyped SNPs in the Han Chinese population (HCB) of the HapMap project (Phase II database) by using a haplotye-tagging SNP approach (Couzin, 2006), with a minor allele frequency $\geq 5 \%$. The selected htSNPs had an estimated correlation coefficient $\left(\mathrm{r}^{2}\right)$ of $>0.8$. Candidate SNP rs11099592, as a non-synonymous coding SNP, was selected based on previously reported associations with he-
Table 1 - Selected clinical and demographic characteristics of the subjects in the case and control population.

\begin{tabular}{|c|c|c|c|}
\hline Variable & $\begin{array}{c}\text { Patients with } \mathrm{HCC} \\
(\mathrm{n}=400)\end{array}$ & $\begin{array}{l}\text { Controls } \\
(\mathrm{n}=480)\end{array}$ & $\mathrm{p}$ \\
\hline \multicolumn{4}{|l|}{ Age, years } \\
\hline Mean (SD) & $49.3(12.0)$ & $48.6(10.0)$ & $0.342^{\dagger}$ \\
\hline$\leq 49, \mathrm{n}(\%)$ & $201(50.3)$ & $264(55.0)$ & $0.16^{\ddagger}$ \\
\hline$>49, \mathrm{n}(\%)$ & $199(49.7)$ & $216(45.0)$ & \\
\hline \multicolumn{4}{|l|}{ Sex, n (\%) } \\
\hline Male & $347(86.7)$ & $384(80.0)$ & $0.008^{\ddagger}$ \\
\hline Female & $53(13.3)$ & $96(20.0)$ & \\
\hline \multicolumn{4}{|c|}{ Nationality, n (\%) } \\
\hline Han & $256(64.0)$ & $359(74.8)$ & $0.001^{\ddagger}$ \\
\hline non-Han ${ }^{\mathrm{a}}$ & $144(36.0)$ & $121(25.2)$ & \\
\hline \multicolumn{4}{|c|}{ Smoking status, n (\%) } \\
\hline Smoker & $162(40.5)$ & $152(31.7)$ & $0.006^{\star}$ \\
\hline Nonsmoker & $238(59.5)$ & $328(68.3)$ & \\
\hline \multicolumn{4}{|c|}{ Smoking level, pack-years } \\
\hline Mean (SD) & $22.1(14.1)$ & $19.1(15.9)$ & $0.08^{\dagger}$ \\
\hline$\leq 23, \mathrm{n}(\%)$ & $102(63.0)$ & $100(65.8)$ & $0.60^{\ddagger}$ \\
\hline$>23, \mathrm{n}(\%)$ & $60(37.0)$ & $52(34.2)$ & \\
\hline \multicolumn{4}{|c|}{ Alcohol use, n (\%) } \\
\hline Drinker & $116(29.0)$ & $145(30.2)$ & $0.696^{\ddagger}$ \\
\hline Nondrinker & $284(71.0)$ & $335(69.8)$ & \\
\hline \multicolumn{4}{|c|}{ HBV carriers, n (\%) } \\
\hline Yes & $303(75.7)$ & $186(38.8)$ & $<.001^{\ddagger}$ \\
\hline No & $97(24.3)$ & $294(61.2)$ & \\
\hline \multicolumn{4}{|c|}{ First-degree family history of $\mathrm{HCC}, \mathrm{n}(\%)$} \\
\hline Positive & $74(18.5)$ & $26(5.4)$ & $<.001^{+}$ \\
\hline Negative & $326(81.5)$ & $454(94.6)$ & \\
\hline
\end{tabular}

${ }^{a}$ Non-Han individuals included Zhuang $(\mathrm{n}=139)$, Jing $(\mathrm{n}=1)$ and Yao $(\mathrm{n}$ $=4$ ) nationalities. In the controls, all of the non-Han individuals were of the Zhuang nationality $(\mathrm{n}=121)$. HBV - hepatitis B virus, HCC hepatocellular carcinoma, $\mathrm{SD}$ - standard deviation. ${ }^{\dagger} P$ value for two-tailed $t$-test. ${ }^{\ddagger} P$ value for two-tailed $\chi^{2}$ test.

matological malignancies (Ostrovsky et al., 2007). Six htSNPs across the 4.9-kb region spanning HPSE loci on chromosome 4q21.3 (84430497-84480330; NCBI Build 37 , hg18), from $5 \mathrm{~kb}$ upstream to $5 \mathrm{~kb}$ downstream of HPSE, were selected for genotyping. These tagging polymorphisms were rs4328905 (intron2), rs4693608 (intron3), rs11099592 (exon8), rs4364254 (intron10), rs12331678 (intron10) and rs12503843 (intron12), which were distributed throughout the full length of the gene. rs12331678 and rs 4364254 could not be included in the blocks. The linkage disequilibrium (LD) plot for the full HPSE gene is shown in Figure 1.

\section{Genotyping}

Genomic DNA from peripheral blood leukocytes was extracted from $5 \mathrm{~mL}$ of whole blood by using standard phenol/chloroform protocols. DNA samples were diluted to 10 


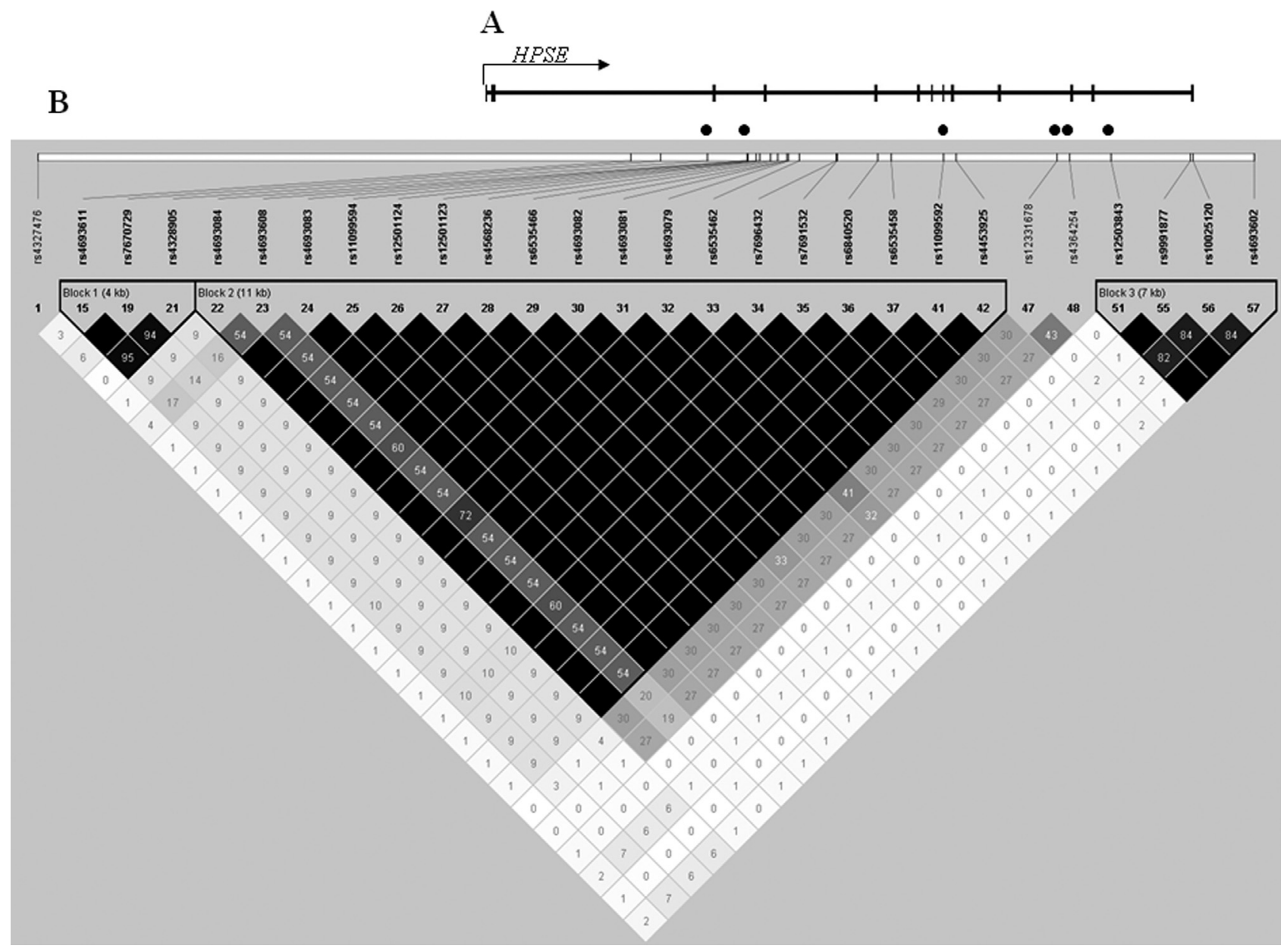

Figure 1 - SNPs in the region of HPSE located on chromosome 4q21.3. (A) HPSE gene structure. Vertical bars represent the 13 exons (5' to 3'). The arrow shows the transcription start site and direction of gene transcription. Solid black points indicate the location of htSNPs in the gene. (B) Diagram of the block structure of HPSE on chromosome 4q21.3 generated with Haploview software. The value within each diamond represents the pairwise correlation between tagging SNPs (measured as D') defined by the upper left and the upper right sides of the diamond. The strength of the linkage disequilibrium (LD) is shown in increasing shades of gray. The diamonds without a number correspond to D' $=1$. Shading represents the magnitude and significance of pairwise LD. Selected htSNPs in three major blocks of HPSE are shown.

$\mathrm{ng} / \mu \mathrm{L}$ and distributed onto 96-well plates, each of which contained 94 samples and two wells of no DNA-control water.

The six htSNPs in HPSE were genotyped in our case-control populations using a PCR-RFLP (polymerase chain reaction-restriction fragment length polymorphism) assay. The PCR fragments covering the relevant htSNPs were amplified via PCR from genomic DNA and the amplicons then digested with an appropriate restriction enzyme that specifically cleaved one allele. The digestion products were subjected to gel electrophoresis and visualized under UV light via Goldview staining. The PCR primers used in the PCR-RFLP assays and the appropriate restriction enzymes are shown in Table 2. Genotyping was done by staff members who were blinded to the subjects' case or control status. The accuracy of the genotyping data for each polymorphism obtained via the PCR-RFLP analyses was validated by the direct sequencing of a $15 \%$ masked random sample of cases and controls, and all of the results showed $100 \%$ concordance.

\section{Statistical analysis}

Genotype and allele frequencies for HPSE polymorphisms were determined by gene counting. The degree of fit to Hardy-Weinberg equilibrium was tested using the $\chi^{2}$ test. Multiple logistic regression analyses were used to evaluate the associations between the polymorphisms and the risk of HCC and were adjusted to account for confounding factors (including age, sex, smoking and drinking status, and level of smoking); the $\mathrm{p}$ values, odds ratios (ORs) and $95 \%$ confidence intervals (95\% CIs) were then calculated. Potential modification of the effect of the polymorphisms on the risk of HCC by the possible confounding factors was assessed by adding interaction terms to the logistic model and by stratification analyses of subgroups of subjects determined by these factors. All statistical analyses were done with SPSS software (version 10.0, SPSS 


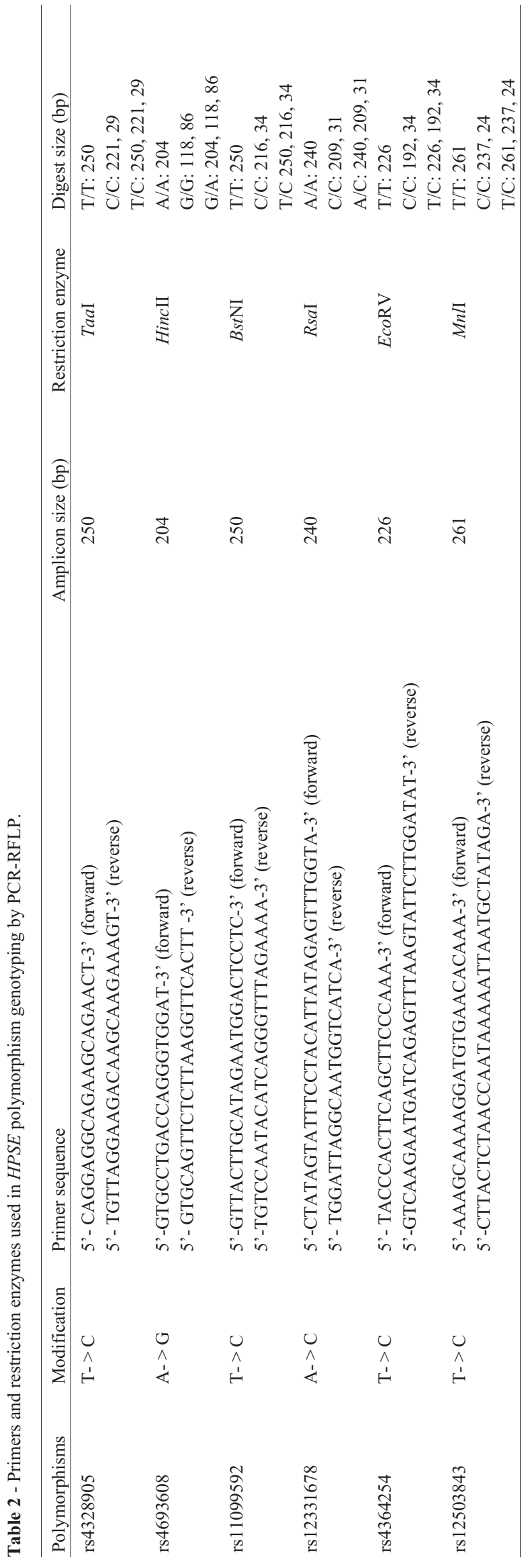

Inc.). SNP spectral decomposition was used to calculate the $\mathrm{M}_{\text {eff }}$ value and thus correct for multiple testing (an LDbased method, available at http://gump.qimr.edu.au/general/daleN/SNPSpD/). This correction strategy accounted for the LD between polymorphic sites. Since failure to account for the non-independence of SNPs would make the Bonferroni correction over-conservative, a value of $p<$ $0.01(0.05 / 5)$ was considered to be statistically significant (Nyholt, 2004).

\section{Results}

Table 1 compares the characteristics of the cases and controls. The two groups were comparable with regard to age, drinking status and pack-years of smoking $(\mathrm{p}>0.05)$. However, compared with controls, there were more men ( $\mathrm{p}$ $=0.008)$, smokers $(p=0.006)$, HBV carriers $(p=0.001)$ and patients with a history of HCC among their first-degree biological relatives $(\mathrm{p}=0.001)$ in the cases group.

The genotype and allele distributions of the six htSNPs between patients with HCC and the controls are summarized in Table 3. The genotype distributions for the six htSNPs were in Hardy-Weinberg equilibrium in each group $(p=0.05)$. Significant correlations were found between the SNPs rs 12331678 and rs 12503843 and the risk of HCC in the overall samples (adjusted OR, 1.69; 95\% CI, $1.17-2.43 ; \mathrm{p}=0.0046$; adjusted OR, $1.52 ; 95 \% \mathrm{CI}$, $1.13-2.05 ; \mathrm{p}=0.0055)$ in the log-additive model. The level of significance was maintained after correcting for multiple testing (SNPSpD). When the analyses were stratified by HBV status, the SNP rs 12503843 was significantly associated with the susceptibility to HCC in HBV carriers $(\mathrm{p}=$ 0.030 ) in the log-additive model. There were associations between rs 12331678 and the risk of HCC in non-HBV carriers (adjusted OR, 2.19; 95\% CI, 1.25-3.85; $\mathrm{p}=0.0074$ ) in the log-additive model, but no associations were found in HBV carriers. An elevated frequency of the A allele for rs12331678 was observed in patients with HCC. The A allele frequency was significantly higher in patients than in controls $(0.11$ vs. $0.074, \mathrm{p}=0.006)$. The rs $12503843-\mathrm{T}$ allele may be also a risk factor for HCC (0.16 vs. $0.11, \mathrm{p}=$ 0.003). Subjects bearing the rs12331678-A or rs12503843-T allele had an increased risk of HCC (Table 4).

In a multiple logistic regression analysis, rs 12331678 and rs 12503843 were significantly associated with HCC when adjusted for the effect of rs 12503843 and rs 12331678 (residual $\mathrm{p}=0.011$ and 0.0062 respectively; Table 5).

\section{Discussion}

Our study comprehensively assessed common variations in the HPSE region that were excluded in our GWAS study (Zhang et al., 2010). In the present study, we found that two polymorphisms in the HPSE gene, rs 12331678 in intron 10 and rs12503854 in intron 12, were significantly 


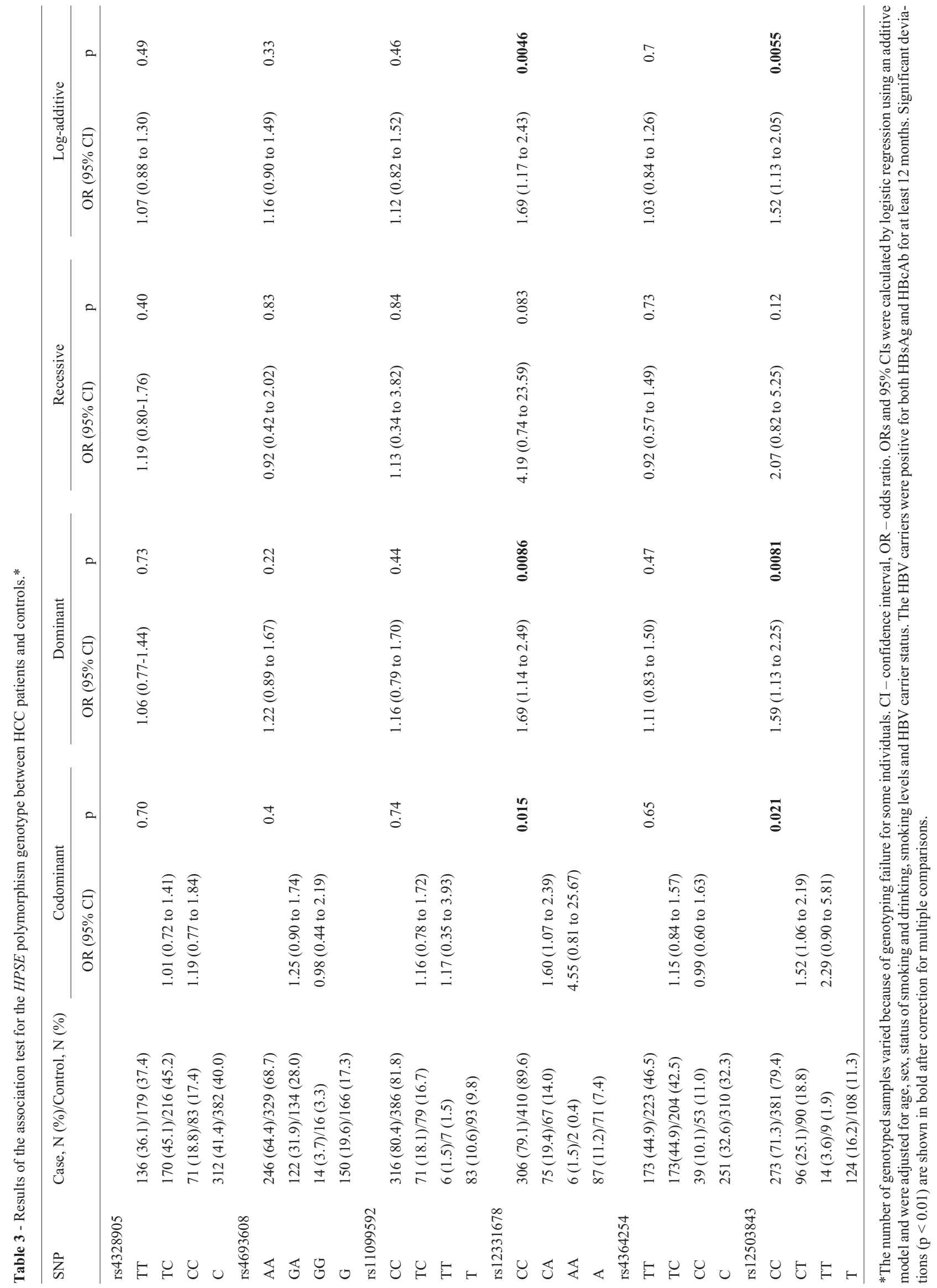




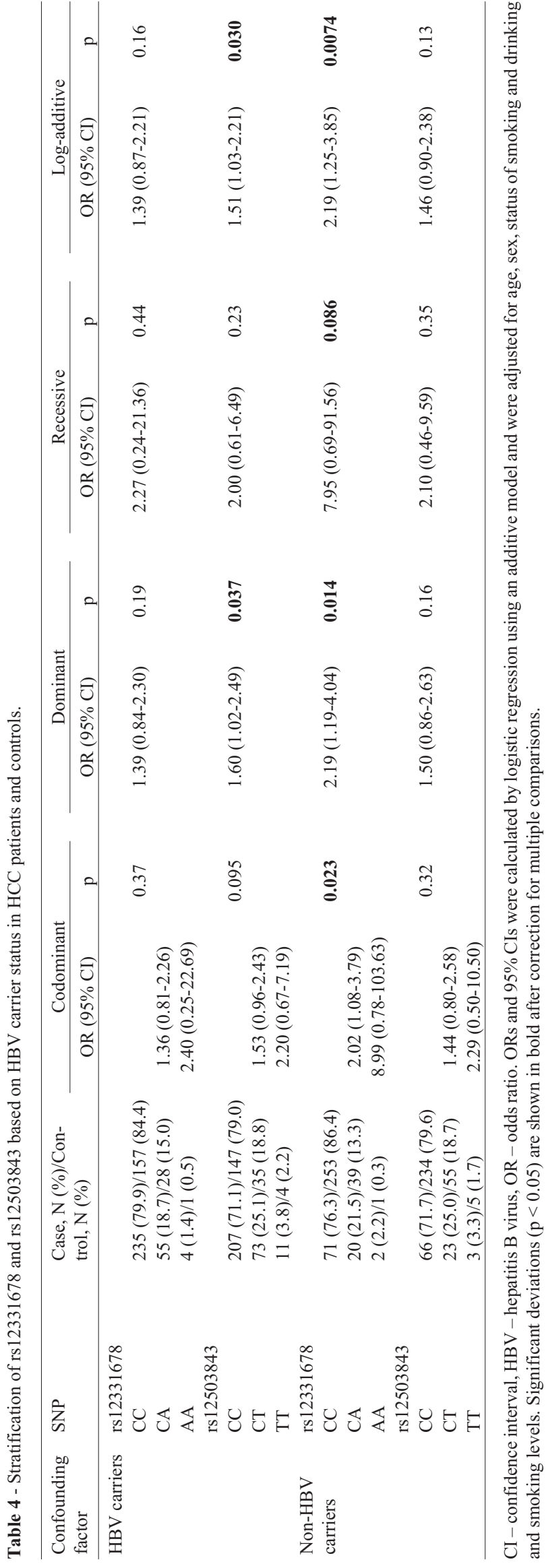

associated with susceptibility to HCC in a Chinese population. The associations between SNPs and risk of HCC were further examined with stratification based on HBV carrier status. There was a significant interaction between rs12503843 and HBV carrier status, suggesting that this status modified the susceptibility to HCC related to the rs12503843 genotype. There was also a significant association between rs 12331678 and risk of HCC in non-HBV carriers. Conditional logistic regression analysis for the independent effect of one significant SNP adjusted by the other SNP suggested that rs 12331678 or rs12503843 contributed an independent effect to the significant association with the risk of HCC, respectively. These findings confirmed the initial hypothesis that the HPSE gene may play an important role in the pathogenesis of HCC.

The current view is that some functionally important non-coding variants may qualitatively or quantitatively alter gene expression (Wang et al., 2006). The polymorphism rs12331678 in intron 10, located at a block-block boundary, showed weak linkage disequilibrium with the other variants in blocks 2 and 3 . This result suggests the polymorphism rs 12331678 may be a causative variant. Indeed, the type of allele $(\mathrm{C}$ or $\mathrm{A})$ predicts the changes in regulatory function for loci in terms of the transcription of HPSE. Bioinformatics prediction of the change in transcriptional factors was done using Alibaba2.1 software. Risk allele rs 12331678 [A] can bind the factor SP1 (specificity protein 1), but the $\mathrm{C}$ allele does not. SP1, a nuclear transcription factor, plays an extremely important role in the growth and metastasis of many tumors, including HCC, by regulating growth-related signal transduction, angiogenesis-related pathways and other factors. Thus, the alleles of rs 12331678 may be involved in the regulation of HPSE expression, although the potential functional roles of this SNP remain to be fully elucidated.

The potential mechanisms involved in the association between rs12503843 and the risk of HCC may include the ability of this SNP to exert a functional role and to act as a marker in tight LD with other functional SNPs in the HPSE 3'UTR. Ostrovsky et al. (2007) have shown that SNP rs4693602, which maps to a distal part of the 3'-UTR of the HPSE gene, was associated with multiple myeloma (MM) and may modify HSPE expression. The intronic polymorphism rs 12503843 is in tight LD with rs4693602 (see Figure 1) and hence the intron 12 SNP might act as a genetic marker, possibly because it too is in tight LD with another SNP downstream in the HPSE 3'-UTR region.

SNP rs11099592 is an A-G replacement located in the coding region of HPSE (exon 8) that results in the substitution of arginine for lysine at position 307 . In contrast to the findings of Ostrovsky et al. (2007), no association was observed here between the SNP rs11099592 and the risk of $\mathrm{HCC}$ in the log-additive model (adjusted OR, 1.12; 95\% CI, $0.82-1.52 ; \mathrm{p}=0.46$ ), a finding consistent with the results of Winter et al. (2008). However, arginine (rs11099592-A) 
Table 5 - Independent effects of rs12331678 and rs12503843 in the overall populations.

\begin{tabular}{|c|c|c|c|c|c|c|}
\hline Polymorphisms & Cases, n (\%) & Controls, n (\%) & OR $(95 \% \mathrm{CI})^{\mathrm{a}}$ & $\mathrm{p}^{a}$ & OR $(95 \% \mathrm{CI})^{\mathrm{b}}$ & $\mathrm{p}^{b}$ \\
\hline \multicolumn{7}{|l|}{ rs 12331678} \\
\hline $\mathrm{C} / \mathrm{C}$ & $300(79.4)$ & $410(85.6)$ & $1.62(1.16-2.26)$ & 0.0042 & $1.55(1.10-2.17)$ & 0.011 \\
\hline $\mathrm{A} / \mathrm{C}$ & $72(19.1)$ & $67(14)$ & & & & \\
\hline $\mathrm{A} / \mathrm{A}$ & $6(1.6)$ & $2(0.4)$ & & & & \\
\hline \multicolumn{7}{|l|}{ rs 12503843} \\
\hline $\mathrm{C} / \mathrm{C}$ & $271(71.7)$ & $381(79.5)$ & $1.53(1.16-2.01)$ & 0.0023 & $1.47(1.12-1.95)$ & 0.0062 \\
\hline $\mathrm{T} / \mathrm{C}$ & 94 (24.9) & 89 (18.6) & & & & \\
\hline $\mathrm{T} / \mathrm{T}$ & $13(3.4)$ & $9(1.9)$ & & & & \\
\hline
\end{tabular}

CI - confidence interval, OR - odds ratio. The $\mathrm{p}$ values, ORs and $95 \%$ CIs were calculated by logistic regression using an additive model and were adjusted for age, sex, status of smoking and drinking, smoking levels and HBV carrier status. ${ }^{\mathrm{a}, \mathrm{b}}$ Values before $\left({ }^{\mathrm{a}}\right)$ and after $\left({ }^{\mathrm{b}}\right)$ adjustment for rs 12503843 or rs12331678. Significant deviations $(\mathrm{p}<0.05)$ are shown in bold.

and lysine (rs11099592-G) are basic amino acids, and this type of modification might not cause an obvious functional distortion of activated heparanase; this situation may have contributed to the lack of an association between rs1 1099592 and HCC. Another possible explanation is that different diseases and population-specific variations may involve different genetic mechanisms of susceptibility.

Previous work suggested that rs4693608, as a functional polymorphism, was strongly correlated with the risk of acute graft-versus-host disease (GVHD) (Ostrovsky et al., 2013). However, our negative results could be explained by differences in the genetic effect among ethnic groups, e.g., population differences in the LD pattern or allele frequencies of HPSE. For example, there may be a small, population-specific effect of HPSE rs4693608 on the development of HCC. Indeed, the allelic and genotypic frequencies of HPSE rs4693608 vary with ethnicity. For instance, the frequencies of the rs $4693608 \mathrm{G}$ allele and GG genotype were 0.17 and 0.03 , respectively, in our study with 480 controls compared to 0.46 and 0.17 in Israeli (Ostrovsky et al., 2014). Ethnic variation in the distribution of the HPSE genotype warrants additional comparative studies in other populations of different ancestry, such as Caucasians and Israelis, to confirm our results.

rs4328905 located in intron 2 of HPSE showed no association with the risk of HCC in our study. Likewise, Yue et al. (2010) and Li et al. (2012) reported no association between rs4328905 and the risk of gastric cancer. Together, these findings indicate that rs 4328905 is not correlated with the incidence of cancers such as HCC and gastric cancer. Similarly, the association between rs4364254 and the risk of HCC risk was not significant in the present study. Ostrovsky et al. (2010) provided the first evidence of a correlation between rs4364254 and the risk of GVHD in a recessive model. In addition, individuals with rs4364254-TT possessed relatively high mRNA levels $(\mathrm{p}=0.0029)$ (Ostrovsky et al., 2009) and there was a significant correlation with poor survival ( $\mathrm{p}=0.013$ ) (Li et al., 2012). These apparent discrepancies may be related to differences among diseases and the relatively low number of patients included in the studies.

Our data revealed two putative HPSE SNPs associated with a risk of HCC. However, several limitations of this study should be noted First, as a hospital-based study, our HCC cases were selected from hospitals whereas the controls were selected from a community population so that inherent selection bias cannot be completely excluded. With further adjustment and stratification in the data analyses, potential confounding factors could have been minimized. Second, although the highly significant association between HPSE and the risk of HCC was derived from a biologically-based a priori hypothesis, our initial findings need to be verified independently in other populations with high incidences of HCC, such as other southern Chinese, as well as Singaporeans and Taiwanese. Lastly, the role of intronic SNPs should be taken into account.

In conclusion, we evaluated the relationship between HPSE gene polymorphisms and susceptibility to HCC in a southern Chinese population. Two intronic SNPs were found to be correlated with a risk of HCC, but the molecular mechanisms linking these noncoding variants with $\mathrm{HCC}$ are still unclear. Direct connection between HCC-associated variants and heparanase expression or function should be explored in future studies.

\section{Acknowledgments}

The authors thank all the individuals tested in this study, their families and the collaborating clinicians for their participation. This study was partly supported by grants from the Chinese National High-tech Program (grant no. 2006AA02A412) and the Open Project of the State Key Laboratory of Proteomics (grant no. SKLP-O200904).

\section{References}

Bishop JR, Schuksz M and Esko JD (2007) Heparan sulphate proteoglycans fine-tune mammalian physiology. Nature 446:1030-1037. 
Chen G, Dang YW, Luo DZ, Feng ZB and Tang XL (2008) Expression of heparanase in hepatocellular carcinoma has prognostic significance: a tissue microarray study. Oncol Res 17:183-189.

Chen XP, Liu YB, Rui J, Peng SY, Peng CH, Zhou ZY, Shi LH, Shen HW and Xu B (2004) Heparanase mRNA expression and point mutation in hepatocellular carcinoma. World $\mathrm{J}$ Gastroenterol 10:2795-2799.

Couzin J (2006) Genomics. The HapMap gold rush: researchers mine a rich deposit. Science 312:1131.

El-Assal ON, Yamanoi A, Ono T, Kohno H and Nagasue N (2001) The clinicopathological significance of heparanase and basic fibroblast growth factor expressions in hepatocellular carcinoma. Clin Cancer Res 7:1299-1305.

Ferlay J, Shin HR, Bray F, Forman D, Mathers C and Parkin DM (2010) Estimates of worldwide burden of cancer in 2008: GLOBOCAN 2008. Int J Cancer 127:2893-2917.

Goldshmidt O, Yeikilis R, Mawasi N, Paizi M, Gan N, Ilan N, Pappo O, Vlodavsky I and Spira G (2004) Heparanase expression during normal liver development and following partial hepatectomy. J Pathol 203:594-602.

Huang GL, Li BK, Zhang MY, Wei RR, Yuan YF, Shi M, Chen XQ, Huang L, Zhang HZ and Liu W (2012) Allele loss and down-regulation of heparanase gene are associated with the progression and poor prognosis of hepatocellular carcinoma. PLoS One 7:e44061.

Li AL, Song YX, Wang ZN, Gao P, Miao Y, Zhu JL, Yue ZY and $\mathrm{Xu}$ HM (2012) Polymorphisms and a haplotype in heparanase gene associations with the progression and prognosis of gastric cancer in a northern Chinese population. PLoS One 7:e30277.

Liu YB, Gao SL, Chen XP, Peng SY, Fang HQ, Wu YL, Peng CH, Tang Z, Xu B and Wang JW (2005) Expression and significance of heparanase and $\mathrm{nm} 23-\mathrm{H} 1$ in hepatocellular carcinoma. World J Gastroenterol 11:1378-1381.

Nyholt DR (2004) A simple correction for multiple testing for single-nucleotide polymorphisms in linkage disequilibrium with each other. Am J Hum Genet 74:765-769.

Ostrovsky O, Korostishevsky M, Levite I, Leiba M, Galski H, Vlodavsky I and Nagler A (2007) Association of heparanase gene (HPSE) single nucleotide polymorphisms with hematological malignancies. Leukemia 21:2296-2303.

Ostrovsky O, Korostishevsky M, Shafat I, Mayorov M, Ilan N, Vlodavsky I and Nagler A (2009) Inverse correlation between HPSE gene single nucleotide polymorphisms and heparanase expression: possibility of multiple levels of heparanase regulation. J Leukoc Biol 86:445-455.

Ostrovsky O, Shimoni A, Rand A, Vlodavsky I and Nagler A (2010) Genetic variations in the heparanase gene (HPSE) associate with increased risk of GVHD following allogeneic stem cell transplantation: effect of discrepancy between recipients and donors. Blood 115:2319-2328.

Ostrovsky O, Shimoni A, Baryakh P, Morgulis Y, Mayorov M, Beider K, Shteingauz A, Ilan N, Vlodavsky I and Nagler A (2014) Modification of heparanase gene expression in response to conditioning and LPS treatment: strong correlation to rs4693608 SNP. J Leukoc Biol 95:677-688.
Ralph S, Brenchley PE, Summers A, Rosa DD, Swindell R and Jayson GC (2007) Heparanase gene haplotype (CGC) is associated with stage of disease in patients with ovarian carcinoma. Cancer Sci 98:844-849.

Sasisekharan R, Shriver Z, Venkataraman G and Narayanasami U (2002) Roles of heparan-sulphate glycosaminoglycans in cancer. Nat Rev Cancer 2:521-528.

Schafer DF and Sorrell MF (1999) Hepatocellular carcinoma. Lancet 353:1253-1257.

Wang GJ, Yang P and Xie HG (2006) Gene variants in noncoding regions and their possible consequences. Pharmacogenomics 7:203-209.

Winter PC, McMullin MF and Catherwood MA (2008) Lack of association of the heparanase gene single-nucleotide polymorphism Arg307Lys with acute lymphoblastic leukaemia in patients from Northern Ireland. Leukemia 22:1629-1631, 1631-1633.

Xiao Y, Kleeff J, Shi X, Buchler MW and Friess H (2003) Heparanase expression in hepatocellular carcinoma and the cirrhotic liver. Hepatol Res 26:192-198.

Yang JM, Wang HJ, Du L, Han XM, Ye ZY, Fang Y, Tao HQ, Zhao ZS and Zhou YL (2009) Screening and identification of novel $\mathrm{B}$ cell epitopes in human heparanase and their anti-invasion property for hepatocellular carcinoma. Cancer Immunol Immunother 58:1387-1396.

Yue Z, Song Y, Wang Z, Luo Y, Jiang L, Xing L, Xu H and Zhang X (2010) Association of heparanase gene (HPSE-1) single nucleotide polymorphisms with gastric cancer. J Surg Oncol 102:68-72.

Zhai Y, Zhou G, Deng G, Xie W, Dong X, Zhang X, Yu L, Yang H, Yuan X and Zhang H (2006) Estrogen receptor alpha polymorphisms associated with susceptibility to hepatocellular carcinoma in hepatitis B virus carriers. Gastroenterology 130:2001-2009.

Zhai Y, Qiu W, Dong XJ, Zhang XM, Xie WM, Zhang HX, Yuan XY, Zhou GQ and He FC (2007) Functional polymorphisms in the promoters of MMP-1, MMP-2, MMP-3, MMP-9, MMP-12 and MMP-13 are not associated with hepatocellular carcinoma risk. Gut 56:445-447.

Zhang H, Zhai Y, Hu Z, Wu C, Qian J, Jia W, Ma F, Huang W, Yu $\mathrm{L}$ and Yue W (2010) Genome-wide association study identifies 1p36.22 as a new susceptibility locus for hepatocellular carcinoma in chronic hepatitis B virus carriers. Nat Genet 42:755-758.

Zhang Y, Li L, Wang Y, Zhang J, Wei G, Sun Y and Shen F (2007) Downregulating the expression of heparanase inhibits the invasion, angiogenesis and metastasis of human hepatocellular carcinoma. Biochem Biophys Res Commun 358:124-129.

\section{Internet Resources}

Alibaba2.1 software, http://www.gene-regulation.com/pub/programs/alibaba2.

Associate Editor: Maria Rita Passos-Bueno

License information: This is an open-access article distributed under the terms of the Creative Commons Attribution License (type CC-BY), which permits unrestricted use, distribution and reproduction in any medium, provided the original article is properly cited. 